\title{
Pesquisas Denominadas Estado da Arte sobre Avaliação e Matemática na Educação Infantil
}

\author{
Investigaciones Denominadas Estado del Arte sobre Evaluación y
}

Matemática en la Educación Infantil

Research Denominated State of Art on Evaluation and Mathematics in

\author{
Child Education
}

\author{
Crislaine de Anunciação Roveda ${ }^{1}$ \\ Fabrício Monte Freitas ${ }^{2}$ \\ João Alberto da Silva ${ }^{3}$ \\ Paola Reyer Marques ${ }^{4}$
}

\begin{abstract}
Resumo
Este trabalho consiste em um levantamento de produções científicas nacionais produzidas nos últimos cinco anos, a fim de identificar o que vem sendo produzido referente ao ensino das crianças na etapa da Pré-Escola. Os procedimentos metodológicos consistiram em uma análise no banco de dados (2012 - 2016) do Seminário Internacional de Pesquisa em Educação Matemática, Encontro Nacional de Educação Matemática, Boletim de Educação Matemática, Associação Nacional de Pós-Graduação e Pesquisa em Educação e Biblioteca Digital Brasileira de Teses e Dissertações. O corpus inicial do estudo constitui-se de 2196 trabalhos, após a realização de três triagens, encontramos o corpus de análise, constituído de 24 trabalhos que comtemplaram cinco temáticas: Avaliação, Conteúdos de Aprendizagem; Desempenho do aluno, Pesquisas bibliográficas e Estados da Arte e Práticas Pedagógicas. Direcionamos nosso estudo nas contribuições da avaliação matemática na Educação Infantil, nossa análise restringiu-se a quatro produções, organizadas nas categorias Avaliação na Educação Infantil, discutida através da utilização de relatórios e teste psicológico, apontando limites e possibilidades desses instrumentos e Desempenho do aluno na Educação Infantil, constatando-se que há uma influência da etapa da Educação Infantil no processo de aprendizagem do $1^{\circ}$ ano do Ensino Fundamental, onde prioriza-se o ensino das linguagens, oral e escrita em relação as habilidades Matemáticas. Nas duas categorias, verifica-se que a avaliação nessa etapa de ensino abrange um caráter prevalentemente formativo. $\mathrm{O}$ estudo revelou que essa temática é ainda incipiente, o que reforça a necessidade de aprofundar estudos.
\end{abstract}

Palavras-chaves: Avaliação; Educação Infantil; Pré-Escola; Matemática.

\section{Resumen}

Este trabajo consiste en un levantamiento de producciones científicas nacionales producidas en los últimos cinco años, a fin de identificar lo que viene siendo producido referente a la enseñanza de los niños en la etapa de la Pre-Escuela. Los procedimientos metodológicos consistieron en un análisis en el banco de datos (2012 2016) del Seminario Internacional de Investigación en Educación Matemática, Encuentro Nacional de

\footnotetext{
${ }^{1}$ Mestranda em Educação em Ciências: Química da Vida e Saúde; Universidade Federal do Rio Grande FURG; Rio Grande; Rio Grande do Sul; Brasil; crislaine@ furg.br.

2 Doutorando em Educação em Ciência: Química da Vida e Saúde; Universidade Federal do Rio Grande FURG; Rio Grande; Rio Grande do Sul; Brasil; fmfreitas86@ hotmail.com. Orientador do trabalho.

3 Doutor em Educação; Universidade Federal de Rio Grande; Rio Grande, Rio Grande do Sul, Brasil; joaosilva@furg.br.

${ }_{4}^{4}$ Doutoranda em Educação em Ciência: Química da Vida e Saúde; Universidade Federal do Rio Grande FURG; Rio Grande; Rio Grande do Sul; Brasil; paolareyer@gmail.com.
} 
Educación Matemática, Boletín de Educación Matemática, Asociación Nacional de Postgrado e Investigación en Educación y Biblioteca Digital Brasileña de Tesis y Disertaciones. El corpus inicial del estudio se constituyó de 2196 trabajos, después de la realización de tres clasificaciones, encontramos el corpus de análisis, constituido de 24 trabajos que comtemplaron cinco temáticas: Evaluación, Contenidos de Aprendizaje; Desempeño del alumno, Investigaciones bibliográficas y Estados del Arte y Prácticas pedagógicas. En el caso de la educación infantil, nuestro análisis se restringió a cuatro producciones, organizadas en las categorías Evaluación en la Educación Infantil, discutida a través de la utilización de informes y test psicológico, apuntando límites y posibilidades de esos instrumentos y Desempeño del alumno en la enseñanza La educación infantil, constatando que hay una influencia de la etapa de la Educación Infantil en el proceso de aprendizaje del $1^{o}$ año de la Enseñanza Fundamental, donde se prioriza la enseñanza de los lenguajes, oral y escrita en relación a las habilidades Matemáticas. En las dos categorías, se verifica que la evaluación en esta etapa de enseñanza abarca un carácter predominante formativo. El estudio reveló que esta temática es aún incipiente, lo que refuerza la necesidad de profundizar en estudios.

Palabras claves: Evaluación; Educación Infantil; Preescolar; Matemáticas.

\begin{abstract}
This work consists of a survey of national scientific productions produced in the last five years, in order to identify what has been produced regarding the teaching of children in the pre-school stage. The methodological procedures consisted of an analysis in the database (2012-2016) of the International Seminar of Research in Mathematical Education, National Meeting of Mathematical Education, Bulletin of Mathematical Education, National Association of Postgraduate and Research in Education and Brazilian Digital Library of Theses and Dissertations. The initial corpus of the study consisted of 2196 papers, after the accomplishment of three screenings, we found the corpus of analysis, consisting of 24 papers that reviewed five themes: Evaluation, Learning Contents; Student performance, Bibliographical research and State of the Art and Pedagogical Practices. We focused our study on the contributions of mathematical evaluation in Early Childhood Education, our analysis was restricted to four productions, organized in the categories of Early Childhood Education, discussed through the use of reports and psychological test, pointing out the limits and possibilities of these instruments. Infantile Education, it being verified that there is an influence of the stage of Infant Education in the process of learning of the 1st year of Elementary School, where the teaching of languages, oral and written in relation to Mathematics skills is prioritized. In both categories, it is verified that the evaluation in this stage of education covers a predominantly formative character. The study revealed that this theme is still incipient, which reinforces the need to deepen studies.
\end{abstract}

Keywords: Evaluation; Child education; Pre-school; Mathematics.

\title{
1. Introdução
}

O presente Estado da Arte, constitui-se em uma pesquisa em bases de dados a fim de realizar um levantamento bibliográfico de produções científicas na área da Educação Infantil relacionados às temáticas da Matemática e da Avaliação. O levantamento bibliográfico foi feito no período de cinco anos com artigos de cinco bases de dados, onde foram realizadas três triagens a fim de delimitar a pesquisa referente às temáticas propostas. Optou-se pelos estudos relacionados a essas temáticas como forma de aprofundamento, para compreender o que relatam as pesquisas científicas sobre a relevância da Matemática no desenvolvimento da criança para o Ensino Fundamental e a como é realizada a avaliação na Educação Infantil.

Ao elaborar o Estado da Arte, buscou-se, de acordo com Ferreira, "trazer em comum o desafio de mapear e de discutir uma certa produção acadêmica em diferentes campos do conhecimento" (2002, p.257). Assim, a pesquisa relacionada ao Estado da Arte está 
relacionada à Educação Infantil, e delimita-se as áreas da Avaliação, aproximando-se mais da temática da Matemática.

\section{Metodologia}

O Estado Arte consistiu em uma análise dos trabalhos científicos publicados nos últimos cinco anos (2012 - 2016) em cinco base de dados: Seminário Internacional de Pesquisa em Educação Matemática (SIPEM), Encontro Nacional de Educação Matemática (ENEM), Boletim de Educação Matemática (Bolema), Associação Nacional de PósGraduação e Pesquisa em Educação (ANPEd) e Biblioteca Digital Brasileira de Teses e Dissertações (BDTD).

Para compor nossa base de dados, escolhemos o ENEM e SIPEM pelo fato de serem eventos promovidos pela Sociedade Brasileira de Educação Matemática. A revista Bolema por ser um periódico nacional avaliado em QUALIS A1 pela Capes; a ANPEd por ter a finalidade de desenvolver a ciência, a educação e a cultura e por fim, a BDTD pois, reúne em um só portal de busca, as teses e dissertações produzidas em todo o País e por brasileiros no exterior.

O quadro 1 mostra o corpus da investigação. Na primeira coluna elencamos as bases pesquisadas, juntamente do total de trabalhos encontrados no período de investigação determinado. O corpus inicial é constituído de 2.196 trabalhos. A tabela apresenta também as três triagens realizadas e a quantidade de trabalhos considerados em cada uma dessas etapas.

Quadro 1: Constituição do Corpus

\begin{tabular}{|l|c|c|c|c|}
\hline \multicolumn{1}{|c|}{ Base de Pesquisa } & Total & $1^{\circ}$ Triagem & $2^{\text {o }}$ Triagem & $3^{{ }^{\circ} \text { Triagem }}$ \\
\hline SIPEM & 32 & 05 & 02 & - \\
\hline ENEM & 1.741 & 23 & 18 & 02 \\
\hline Bolema & 303 & 06 & 05 & 04 \\
\hline BDTD & 14 & 05 & 05 & 03 \\
\hline ANPEd & 106 & 06 & 06 & $\mathbf{2 4}$ \\
\hline TOTAL & $\mathbf{2 . 1 9 6}$ & $\mathbf{4 5}$ & $\mathbf{3 6}$ & \\
\hline
\end{tabular}

Fonte: Elaborado pelos autores (2017)

A primeira triagem foi realizada através da leitura dos títulos e palavras-chaves quando necessário, observando títulos que se relacionavam a nossa investigação. Na segunda triagem, foi realizada a leitura dos resumos dos trabalhos que, pelo título e pelas palavras-chaves 
foram selecionados na primeira triagem. A terceira e última triagem consistiu na leitura completa dos trabalhos, dos quais, os resumos não possuíam todas as informações necessárias para a compreensão da pesquisa. Adotamos o mesmo procedimento das triagens nas cinco bases de dados consideradas.

A primeira base de dados foi realizada nos anais do SIPEM. No V SIPEM, realizado em Petrópolis, RJ no ano de 2012, analisou-se somente o GT08 - Avaliação em Educação Matemática, pois a GT01 não estava ligada à Educação Infantil, somente aos Anos Iniciais. No VI SIPEM em Pirenópolis, GO em 2015, analisou-se o GT01 - Matemática na Educação Infantil e nos Anos Inicias do Ensino Fundamental e o GT08 - Avaliação em Educação Matemática. O montante de artigos dessas duas edições foram de 32 trabalhos. Na primeira triagem, o número foi reduzido para 05 trabalhos. Em uma segunda triagem, após a leitura dos resumos desses 05 artigos, observou-se que dois deles relataram sobre os Anos Iniciais do Ensino Fundamental e um sobre o sexto ano dos Anos Finais, sendo assim, descartados os três. Na terceira triagem, realizou-se a leitura completa dos outros dois trabalhos, pois os resumos não estavam claros, então constatou-se que tratavam sobre a concepção de avaliação de professores na área da Matemática dos Anos Finais. Assim, não foi aproveitado nenhum trabalho das categorias analisadas da SIPEM.

$\mathrm{Na}$ segunda base de dados, temos o ENEM, tendo como base de pesquisa, as duas últimas edições do evento, respectivamente, o XI ENEM em Curitiba, PR no ano de 2013 e o XII ENEM em São Paulo, SP no ano de 2016. A partir da produção presente nessas duas edições do evento, identificamos 1.741 artigos no montante inicial. Ao delimitar a pesquisa, os artigos que remetem à nossa pesquisa, foi realizada a primeira triagem, onde foram selecionados 23 trabalhos. Após a segunda triagem, restaram 18 trabalhos. Na última triagem, foram descartados 03 trabalhos, pois relatam sobre uma oficina de matemática lúdica, para um grupo de 20 crianças, de 6 a 12 anos, ensino da Matemática para alunos de $9^{\circ}$ ano e outro para o Fundamental II, totalizando assim 15 trabalhos relacionados a Educação Infantil.

Ao analisarmos a terceira base de dados, a Revista Bolema: Boletim de Educação Matemática, foi levado em consideração cinco volumes (Vol. 26 a 30) da revista, entre os anos de 2012 a 2016, respectivamente, totalizando um montante de 303 trabalhos em 16 edições. Na primeira triagem, foram considerados 6 títulos. Para a segunda triagem, foi levado em conta 05 trabalhos, e descartado somente 01 , pois no resumo constava que o artigo se tratava dos Anos Iniciais do Ensino Fundamental. Na leitura completa dos 05 artigos, os quais não continham todas as informações que buscávamos nos seus resumos, constatou-se que somente 02 eram pertinentes a pesquisa, pois os outros 03 relatavam sobre a prática dos 
professores com a utilização de signos, a avaliação por portfólio nos Anos Finais e Ensino Médio e o outro da Matemática nos primeiros anos escolares.

$\mathrm{Na}$ pesquisa realizada na quarta base de dados, a Biblioteca Digital de Teses e Dissertações, foram considerados somente os trabalhos referentes a artigos e/ou produções científicas. Foi realizado uma busca avançada utilizando os seguintes descritores: Matemática, Educação Infantil e Avaliação, e ano de publicação entre 2012 e 2016. Nesse primeiro momento foram totalizados um montante de 14 trabalhos. Na primeira triagem foram considerados 05 títulos. A segunda triagem, após a leitura do resumo, o número manteve-se em 05. Após a realização da última triagem, foi descartado apenas 01 trabalho, que no decorrer do texto apresentou-se como referente aos Anos Iniciais do Ensino Fundamental, constituindo assim, 04 trabalhos.

Na última base de dados, a Associação Nacional de Pós-Graduação em Pesquisa em Educação (ANPEd), foram analisadas: $35^{\circ}$ Reunião anual da ANPEd, em Porto de Galinhas, PE no ano de 2012; 36 Reunião nacional da ANPEd, em Goiânia, GO no ano de 2013 e a 37 Reunião nacional da ANPEd, em Florianópolis, SC no ano de 2016. Analisamos as produções científicas dos grupos de trabalho GT07 - Educação de Crianças de 0 a 6 anos e GT19 Educação Matemática, com um total de 106 artigos. Na primeira triagem, esse número reduziu-se a 06. Como nem todos os seis trabalhos possuíam resumo, optou-se por realizar a leitura completa dos seis trabalhos, passando assim a descartar, na terceira triagem, 03 trabalhos que falavam sobre: Formação de professores no Anos Iniciais, avaliação nos Ciclo de Alfabetização e no $6^{\circ}$ ano do Ensino Fundamental.

Dessa forma, do montante inicial de 2.196 trabalhos, a primeira triagem consistiu-se em 45 trabalhos, a segunda em 36 e a terceira e última em 24 trabalhos, constituindo assim o corpus de análise.

\subsection{Categorias de Análise}

A partir do nosso corpus de análise, organizamos as temáticas emersas nas 24 produções científicas em cinco categorias. As categorias foram construídas com a função de facilitar a identificação das áreas em que as produções se concentram. A partir do levantamento preliminar realizado, descrito na metodologia, construímos as seguintes categorias: (1) Avaliação na Educação Infantil, com dois trabalhos; (2) Conteúdos de Aprendizagem, com um trabalho, (3) Desempenho do aluno na Educação Infantil, com dois trabalhos, (4) Pesquisas bibliográficas e Estados da Arte, com três trabalhos e (5) Práticas Pedagógicas, com 16 trabalhos. 
Devido ao nosso campo de pesquisa estar relacionado às avaliações na Educação Infantil com viés na área da Matemática, será pertinente a descrição de duas categorias emersas: (1) Avaliação na Educação Infantil e (3) Desempenho do aluno na Educação Infantil. Essas duas categorias tratam especificamente das situações que queremos abordar e a sua contribuição relacionada à Educação Matemática em crianças em faixa etária da Educação Infantil, que compreende crianças de zero a cinco anos de idade, conforme a Lei de Diretrizes e Bases da Educação Nacional (1996), bem como na forma como essa avaliação implica no desenvolvimento da criança no primeiro ano da Educação Básica. As demais categorias: Conteúdos de Aprendizagem, Pesquisas bibliográficas e Estados da Arte e Práticas Pedagógicas, apesar de trazerem contribuições científicas importantes sobre a Educação Matemática, não delimitam sobre a avaliação.

\subsubsection{Avaliação na Educação Infantil:}

A avaliação na Educação Infantil é um tema ainda pouco presente nas produções científicas da área, como constata Paz $(2005)^{5}$. Nesta categoria encontramos duas produções científicas que tratam da avaliação na Educação Infantil a partir de instrumentos, como o teste psicológico e o utilizo de relatórios.

Neves (2012) faz um debate atual acerca da avaliação no contexto da Educação Infantil. Tal debate refere-se à adoção, pela Secretaria Municipal de Educação do Rio de Janeiro, do teste psicológico estadunidense Ages and Stages Questionnaires - Third Edition (ASQ-3). O teste $A S Q-3$ é uma avaliação externa em larga escala que avalia as crianças, de quatro meses a cinco anos de idade, individualmente em cinco áreas de desenvolvimento: coordenação motora fina, coordenação motora ampla, comunicação, resolução de problemas e habilidades pessoal e social. O teste é composto de cinco perguntas para cada área de desenvolvimento, com o objetivo de detectar atrasos no desenvolvimento infantil o mais rápido possível. O fato do teste medir alguns aspectos do desenvolvimento infantil com base em apenas cinco perguntas é um dos aspectos criticados pelo autor, enfatizando que não existe uma discussão ampla sobre os limites intrínsecos ao próprio instrumento. Na sua discussão é citado diversos exemplos de perguntas presentes no teste e conclui que através dessas perguntas "não é possível avaliar todos os aspectos do desenvolvimento de maneira ampla; as perguntas referem-se a um determinado contexto estadunidense e, portanto, não consideram ou legitimam as variações culturais e várias das perguntas são desprovidas de

\footnotetext{
${ }^{5}$ A partir de análise da produção acadêmica brasileira presente nas reuniões anuais da ANPEd entre 1993 e 2003
} 
sentido" (p. 2-3). O autor destaca que, além deste instrumento não poder avaliar o desenvolvimento do aluno de uma maneira ampla, os professores não são capazes de construir ou discutir nessa avaliação. A adoção do teste como referencial curricular poderia implicar em uma redução das experiências possíveis na educação infantil em função de estímulos e treinamentos de habilidades parciais.

O autor relata uma experiência de avaliação na Educação Infantil em Belo Horizonte. Através de observação, anotações no Diário de Campo, gravações em áudio e vídeo, entrevistas com crianças, professoras e famílias e observações das Reuniões Pedagógicas, o autor buscou compreender quais formas de avaliação aconteceram na turma pesquisada e como essas formas de avaliar as crianças se concretizaram na prática. A pesquisa revelou que a rotina da turma estruturava-se em torno das brincadeiras e rodas de conversa, podendo evidenciar três tipos de avaliação realizadas com as crianças: (1) Avaliações de diversas atividades nas rodas de conversas, com objetivo de construção de histórias do grupo ao longo de todo o ano; (2) Avaliações individuais, verificando a aprendizagem e dando subsídios para os relatórios individuais 2 vezes ao ano; (3) Relatórios individuais escritos no Diário de Classe, objetivando a comunicação com as famílias, 3 vezes ao longo do ano. Essas avaliações serviram como acompanhamento do desenvolvimento da turma e orientação a prática individual da professora, bem como uma relação sistematizada dos processos de ensino aprendizagem da turma em relação às avaliações individuais. A roda de conversa constituiu-se em um espaço de negociação da ordem e da autoridade, estabelecendo assim formas de participação no grupo e a construção coletiva do conhecimento, relacionando-se com a cultura escolar da educação infantil. Esse espaço possibilitou também a avaliação informal do envolvimento das crianças nas atividades. Nos momentos de avaliação individual, solicitou-se o que não foi enfatizado ao longo do ano nas práticas educativas da professora: a escrita do próprio nome; o reconhecimento das letras do alfabeto, dos numerais e das cores; a contagem de materiais. Ou seja, a avaliação individual das crianças não serviu como base para a prática pedagógica. A escrita dos relatórios caracterizou-se na concepção de avaliação formativa, através do qual crianças e professoras reconstruam, em conjunto, suas práticas cotidianas.

Bresciane (2015), em sua pesquisa de mestrado buscou compreender como professores avaliam os progressos das crianças na Educação Infantil. A partir da análise de relatórios de acompanhamento e avaliação de alunos com uma faixa etária de quatro e cinco anos de idade, a autora identifica o que esses revelam sobre as concepções e práticas de avaliação na Educação em uma rede municipal paulista, destacando seis pontos principais: (1) existe uma 
organização curricular comum a todas as escolas, baseada nos Referenciais Nacionais para a Educação Infantil, a partir do qual é realizada a avaliação; (2) todas as escolas produzem o mesmo tipo de registro, seguindo o que é proposto no regimento do município; (3) a avaliação não se relaciona ao contexto educativo ou aprendizagens desenvolvidas pelas crianças; (4) a avaliação foca preferencialmente nos conteúdos dos eixos de Matemática, Linguagem Oral e Escrita e Formação Pessoal e Social; (5) o foco da avaliação são os resultados, relativos aos conteúdos mencionados, e não o processo de aprendizagem e desenvolvimento; (6) as famílias participam somente no final do processo de avaliação, as crianças não participam.

Os relatórios de avaliação serviram como acompanhamento do desenvolvimento da turma e orientação a prática individual da professora. De modo geral, a escrita é pertinente e respeitosa, revelando que a boa comunicação com as famílias é valorizada. Conclui-se que esses relatórios são compreendidos mais como documentos de constatação, já que focam mais nos resultados da criança do que em seu processo. Somente através deles não seria possível ter conclusões sobre o processo de avaliação como um todo, mas sim detectar indícios de que alguns de seus aspectos precisam ser revistos a fim de torná-los mais homogêneos. A autora destaca que não é a "opção pelo relatório como instrumento que vai determinar a qualidade do registro sobre a avaliação, mas a clareza sobre os seus propósitos, os conhecimentos, habilidades e atitudes necessários para a sua elaboração pelas professoras” (p. 90). Como elementos pertinentes de se conhecer, destacam-se conhecimentos sobre a criança e seu desenvolvimento, sobre os fundamentos, princípios e modos de concretização do currículo na Educação Infantil; as habilidades de observar, registrar, refletir, planejar e sintetizar informações, sendo esses recursos necessários para se tecerem os significados da avaliação. As práticas avaliativas devem ser ancoradas nas experiências das crianças e nas práticas que lhe dão contexto, pois são capazes de regulá-las e reorientá-las. Aponta-se para a formação continuada de professores como meio para se realizarem tais tarefas.

\subsubsection{Desempenho do aluno na Educação Infantil:}

Nesta categoria as produções científicas refletem sobre desempenho dos estudantes na passagem da Educação Infantil ao Ensino Fundamental. Observam-se possíveis influências no desempenho cognitivo através de instrumentos voltados para conhecer as habilidades dessa faixa etária.

O estudo realizado por Pereira (2014) partiu de uma inquietação frente a apontamentos em reuniões na Secretaria da Educação, onde gestoras de Ensino Fundamental afirmavam que alunos oriundos de Centros Infantis (Creches) apresentavam mais dificuldades e desempenho 
inferior no $1^{\mathrm{o}}$ ano do Ensino Fundamental, quando comparados aos alunos egressos das Escolas Municipais de Educação Infantil - EMEI (pré-escola). A autora investiga, junto a alunos ingressantes no $1^{\circ}$ ano do Ensino Fundamental oriundos de Centros Infantis e EMEI, se apresentam descompassos no desempenho escolar. A pesquisa consistiu em um estudo comparativo dos resultados de desempenho em avaliações somativas de Língua Portuguesa e Matemática, aplicando-as bimestralmente. O estudo exploratório revelou que os alunos "oriundos dos Centros Infantis se apropriaram da escrita, do letramento e de conhecimentos matemáticos de maneira semelhante, e em alguns casos, até mesmo superior à dos alunos egressos das EMEI" (p.169).

Com resultado diferente do que fora apontado pelos professores e gestores do Ensino Fundamental, o estudo indica três principais causas para explicar a avaliação equivocada: (1) a presença de diferentes concepções de Educação Infantil na rede; (2) a falta de articulação entre a Educação Infantil e o Ensino Fundamental; (3) a falta de qualificação dos profissionais que atuam na Educação Infantil, tanto docentes como gestores. A partir dos dados emersos percebe-se que o problema está na transição do Ensino Infantil para o Ensino Fundamental e que as concepções acerca das finalidades da Educação Infantil são um dos principais pontos de tensões entre esses dois segmentos de Ensino. A falta de articulação pode estar atrelada a inexperiência de novos professores que ingressam na escola. A partir disso, passou-se a trabalhar com os gestores para uma união desses aspectos, e posteriormente com os professores através de formações continuadas, com o intuito da passagem da Educação Infantil para o Ensino Fundamental não sofresse rupturas.

Pazeto (2016) observa que a avaliação das habilidades cognitivas das crianças, principalmente na pré-escola e nos primeiros anos do Ensino Fundamental, auxilia na compreensão e identificação de quais habilidades estão envolvidas no processo de aprendizagem. A autora realizou seu estudo com o objetivo de verificar se funções executivas, linguagem oral, conhecimentos iniciais sobre linguagem e escrita, variáveis socioeconômicas e biológicas, avaliadas na pré-escola, predizem o desempenho escolar em escrita, leitura e matemática. A investigação partiu de 13 instrumentos para avaliar crianças entre 4 e 6 anos de pré-escola e $1^{\circ}$ ano do Ensino Fundamental, sendo 3 de funções executivas, 3 de linguagem oral, 4 de linguagem escrita, 1 de matemática, 1 ficha de identificação para a família e 1 questionário para os professores.

Destacamos o que Pazeto (2016) traz sobre a avaliação na área da matemática, pois é nosso foco de estudos. Através da elaboração de um teste de aritmética (Seabra et al., 2009, 2013 apud Pazeto, 2016) aplicado somente com alunos do $1^{\circ}$ ano do Ensino Fundamental, o 
estudo buscou avaliar: a capacidade da criança de escrever números por extensos, representados algebricamente de uma sequência de números que o avaliador fala oralmente; escritas de forma crescente ou decrescente; comparação de grandezas numéricas; cálculo de operações que são apresentadas tanto impressas quanto ditadas e por fim, resoluções de problemas escritos. $\mathrm{O}$ escore utilizado foi a quantidade total de acertos na prova. A partir da análise de regressão em árvore, verificou-se que a consciência fonológica por produção oral, conhecimento de letras, leitura e escrita preliminar, vocabulário e dificuldade apontada pelo professor são habilidades preditoras para o desempenho posterior em matemática. Os resultados encontrados corroboram com diversos estudos na área, reforçando a predição dessas habilidades para desempenhos posteriores principalmente na vivência escolar, mostrando-se como área de focus para serem trabalhadas na Educação Infantil.

\section{Conclusões}

A referente pesquisa se propôs a realizar um levantamento bibliográfico ligado à Educação Infantil, abordando principalmente a avaliação em uma perspectiva na área da Matemática. Através da separação por categorias, podemos observar que a produção maior foi na área referente às práticas do ensino, que pode ser tanto na formação do docente atuante nessa área, quanto da própria prática em sala de aula.

Nas áreas descritas nas duas categorias, constatou-se que a avaliação nessa etapa de ensino abrange um caráter prevalentemente formativo. De acordo com Hadji (2001), avaliação formativa é aquela que se situa no centro da ação de formação, proporcionando o levantamento de informações úteis à regulação do processo ensino - aprendizagem, contribuindo para a efetivação da atividade de ensino.

$\mathrm{Na}$ categoria de desempenho, constatou-se que há uma influência da etapa da Educação Infantil no processo de aprendizagem do $1^{\circ}$ ano do Ensino Fundamental, porém, pode se levar em conta, nesses dois trabalhos, que é priorizado o ensino das linguagens, oral e escrita, do que as habilidades na Matemática.

Dessa forma, sente-se a necessidade de abordar conceitos relacionados às formas de avaliação, bem como a importância de abordagens matemáticas para o desenvolvimento da criança na primeira etapa da Educação Básica. 


\section{Referências}

BRASIL. Ministério de Educação e Cultura. Lei de Diretrizes e Bases da Educação no 9394/96, de 20 de dezembro de 1996. Estabelece as Diretrizes e Bases da Educação Nacional. Brasília: MEC, 1996.

BRESCIANE, A. L. A. Avaliação Na Educação Infantil: O Que Nos Revelam Os Relatórios De Um Município Paulista. Pontifícia Universidade Católica de São Paulo, 2015. Disponível em: <https://sapientia.pucsp.br/handle/handle/16208>. Acesso em jun. 2017.

FERREIRA, Norma Sandra de Almeida. As Pesquisas Denominadas "Estado da Arte". Educação \& Sociedade, ano XXIII, no 79, p.257-272, Ago/2002. Disponível em: <http://www.scielo.br/pdf/es/v23n79/10857>. Acesso em mai. 2017.

HADJI, C. A Avaliação desmistificada. Porto Alegre: Artmed Editora, 2001.

NEVES, V. F. A. Avaliação na Educação Infantil: Algumas reflexões. In: Associação Nacional de Pós-Graduação e Pesquisa em Educação, 35., 2012, Porto de galinhas, PE. Anais... Porto de Galinhas: ANPEd, 2012. Disponível em: < http://www.anped.org.br/biblioteca/item/avaliacao-na-educacao-infantil-algumas-reflexoes $>$. Acesso em jun. 2017.

PAZ, S. J. P. A avaliação na educação infantil: análise da produção acadêmica brasileira presente nas reuniões anuais da ANPEd entre 1993 e 2003. Dissertação (Mestrado em Educação). Universidade Federal de Santa Catarina. 2005. Disponível em: < https://repositorio.ufsc.br/handle/123456789/102215>. Acesso em jun. 2017.

PAZETO, T. de C. B. Predição de leitura, escrita e matemática no ensino fundamental por funções executivas, na linguagem oral e habilidades iniciais de linguagem escrita na Educação Infantil. Universidade Presbiteriana Mackenzie, 2016. Disponível em: <http://tede.mackenzie.br/jspui/handle/tede/2899>. Acesso em jun. 2017.

PEREIRA, J. C. S. Educação Infantil no município de Limeira - SP: um estudo comparativo do desempenho de alunos de creches e pré- escolas nos anos iniciais do ensino fundamental. Universidade Federal de Juiz de Fora, 2014. Disponível em: <https://repositorio.ufjf.br/jspui/handle/ufjf/627>. Acesso em jun. 2017. 